\title{
Randomised Trials in Surgery: Problems and Possible Solutions: At last, Sense and Diamonds have been Recovered from an Ocean of Nonsense
}

\author{
Ahmed N Ghanem* \\ Consultant Urologist Surgeon, Egypt \\ *Corresponding author: Ahmed N Ghanem, Consultant Urologist Surgeon, No1 President Mubarak Street, Mansoura 35511, Egypt.. \\ To Cite This Article: Ahmed N Ghanem. Randomised Trials in Surgery: Problems and Possible Solutions: At last, Sense and Diamonds have been \\ Recovered from an Ocean of Nonsense. Am J Biomed Sci \& Res. 2019 - 2(2). AJBSR.MS.ID.000577. DOI: 10.34297/AJBSR.2019.02.000577
}

Received: March 20, 2019 | Published: April 02, 2019

\section{Editorial}

I read with interest this excellent article [1] on which I congratulate and commend the authors for diving deep and wide in order to recover sense and diamonds from an ocean of irrelevant nonsense. I fully support the reported views and recommendations. It is a shame that such landmark articles are not immediately implemented by concerned bodies to become standard law of medical research and advancement, surgical research.

The authors correctly concentrated on problems facing therapeutic surgical studies, and the need to differentiate from drug studies. However, it has perhaps already been realized that prospective observational diagnostic studies do significantly contribute to evidence-based medicine (EBM), with or without randomization or statistics. Such studies also start from the day of encountering the first case, does not require the approval of anybody or committee and only patients' consent is required when and as interference with approved diagnostic or therapeutic procedures is indicated.

Contributions of such diagnostic studies to EBM include, identifying the problems of currently received protocols, proposing new ones with possible novel diagnostic signs or tests and suggestions for resolving problems as demonstrated in the authors' article [1]. Observing new links between known diseases of idiopathic aetiology may reveal new pathos-aetiology of importance in advancing new surgical therapy or modifying old one that had previously proved a failure because of overlooked such pathos-aetiology or incorrect timing of the procedure.

As many have been, and now more than ever might be, fishing for a conflict of interest in such research, please allow me to give them one: it the "type lead poisoning" that inflicts an author to the degree of addiction, causing strong desire to contribute to science advancement and betterment of Global society as charity work. This is not just gas. Some do put their money where their mouth is, costing much of own money, time, efforts and health with no gain whatsoever in terms of career advancement or money making because it is simply of little concern or past it- as it never happened in the past when it was most needed. If, however, such unexpected gain or offer might occur in future it might be welcomed as unconditional grant, mostly spent on advancing current and further future research as judged ethical, appropriate and fit.

\section{References}

1. McCulloch P, Taylor I, Sasako M, Lovett B, Griffin D (2002) Randomised trials in surgery: problems and possible solutions. BMJ 324: 1448-1451. 